ORIGINAL ARTICLE

\title{
The global burden of non-conflict related firearm mortality
}

\section{T S Richmond, R Cheney, C W Schwab}

Injury Prevention 2005;11:348-352. doi: 10.1136/ip.2005.008896

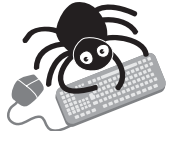

Additional data are available on our website.

See end of article for authors' affiliations

.........................

Correspondence to: Dr T S Richmond, Associate Professor School of Nursing, 412 NEB, University of Pennsylvania, 420 Guardian Drive, Philadelphia, PA 19104, USA; terryr@nursing. upenn.edu

Accepted

7 September 2005
Objective: Understanding global firearm mortality is hindered by data availability, quality, and comparability. This study assesses the adequacy of publicly available data, examines populations for whom firearm mortality data are not publicly available, and estimates the global burden of non-conflict related firearm mortality.

Design: The design is a secondary analysis of existing data. A dataset of countries, populations, economic development, and geographic regions was created, using United Nations 2000 world population data and World Bank classifications of economic development and global regions. Firearm mortality data were obtained from governmental vital statistics reported by the World Health Organization and published survey data. A qualitative review of literature informed estimates for the 15 most populous countries without firearm death data. For countries without data, estimates of firearm deaths were made using quartiles of observed rates and peer reviewed literature.

Main outcome measures: Non-conflict related firearm deaths.

Results: Global non-conflict related firearm deaths were estimated to fall between 196000 and 229000 , adjusted to the year 2000. 162800 firearm deaths adjusted for the year 2000 came from countries reporting data and represent $35 \%$ of the world's 186 countries. Public data are not available for 122 of these 186 countries, representing more than three billion $(54 \%)$ of the world's population, predominately in lower and lower middle income countries. Estimates of firearm death for those countries without data range from 33200 to 66200 .

Conclusions: This study provides evidence that the burden of firearm related mortality poses a substantial threat to local and global health.
$\mathrm{H}$ ealth threats cross national borders and extend beyond infectious disease to include violence. ${ }^{12}$ The World Health Organization (WHO) World Report on Violence and Health (WRVH) urges member nations to examine the impact of intentional injury and develop strategies to reduce violence. $^{3}$ Firearm deaths contribute to this burden of violence. ${ }^{4}$ In some countries, the firearm is the most frequently used weapon for homicide and suicide. ${ }^{56}$ Delineating the burden of firearm violence is hindered by data limitations, with international comparisons heavily weighted toward high income countries with well developed vital statistics systems. ${ }^{78}$ Countries without firearm death data (FDD) are of interest because they account for a large proportion of the world's population.

This study assessed the adequacy of publicly available data, examined populations without FDD, and estimated the global burden of non-conflict related firearm mortality. Conflict related FDD were excluded, not to minimize the importance of conflict related deaths, but to establish a baseline of global firearm mortality, independent of armed conflict.

\section{METHODS}

We compiled a country level dataset of reported FDD from existing public sources, projected these data to the year 2000, assessed and adjusted for missing data on intent, and conducted a literature review to develop estimates of firearm deaths for countries without FDD. These data were compiled for 186 countries with populations greater than 140000 persons. ${ }^{9}$

\section{Data sources}

Firearm deaths by intent for the latest year reported (19942000) were assembled from the WHO-WRVH ${ }^{3}$ and two surveys. ${ }^{6}{ }^{10}$ These data, based on international cause of death coding, exclude military/police action and conflict related firearm deaths. The WHO dataset consists of vital statistics data reported from 100 countries. Country level survey data were obtained from the United Nation's (UN) International Study on Firearm Regulations report and online database, with responses from 69 member nations. ${ }^{10}$ A third data source was a survey of health officials from 36 high/upper middle income countries with populations greater than one million. ${ }^{8}$ With these data we could not adjust for overall underreporting of deaths or age/sex specific rates. For comparability, firearm deaths were projected to UN year 2000 population estimates. ${ }^{9}$

\section{Categories of data availability}

Sources for FDD were prioritized (fig 1). The primary class is the WHO-WRVH data, representing 42 countries with a combined population of 1053658000 . The second data class-survey data with complete intent categories-adds 12 countries with a population of 390438000 . The final data class - survey data with missing intent categories-adds 10 countries with a population of 1342227000 . Total crude firearm death rates (CFDR), percent of intentional injury deaths caused by firearms, and the proportionate share of the world's population were calculated for each class of data availability. Percent of population with FDD and CFDRs were calculated by economic development level and region. We conducted a detailed examination on the 15 most populous countries with FDD and the 15 most populous countries without FDD. These 30 countries account for nearly $80 \%$ of the world's population.

Abbreviations: CFDR, crude firearm death rates; FDD, firearm death data; UN, United Nations; WHO, World Health Organization; WRVH, World Report on Violence and Health. 


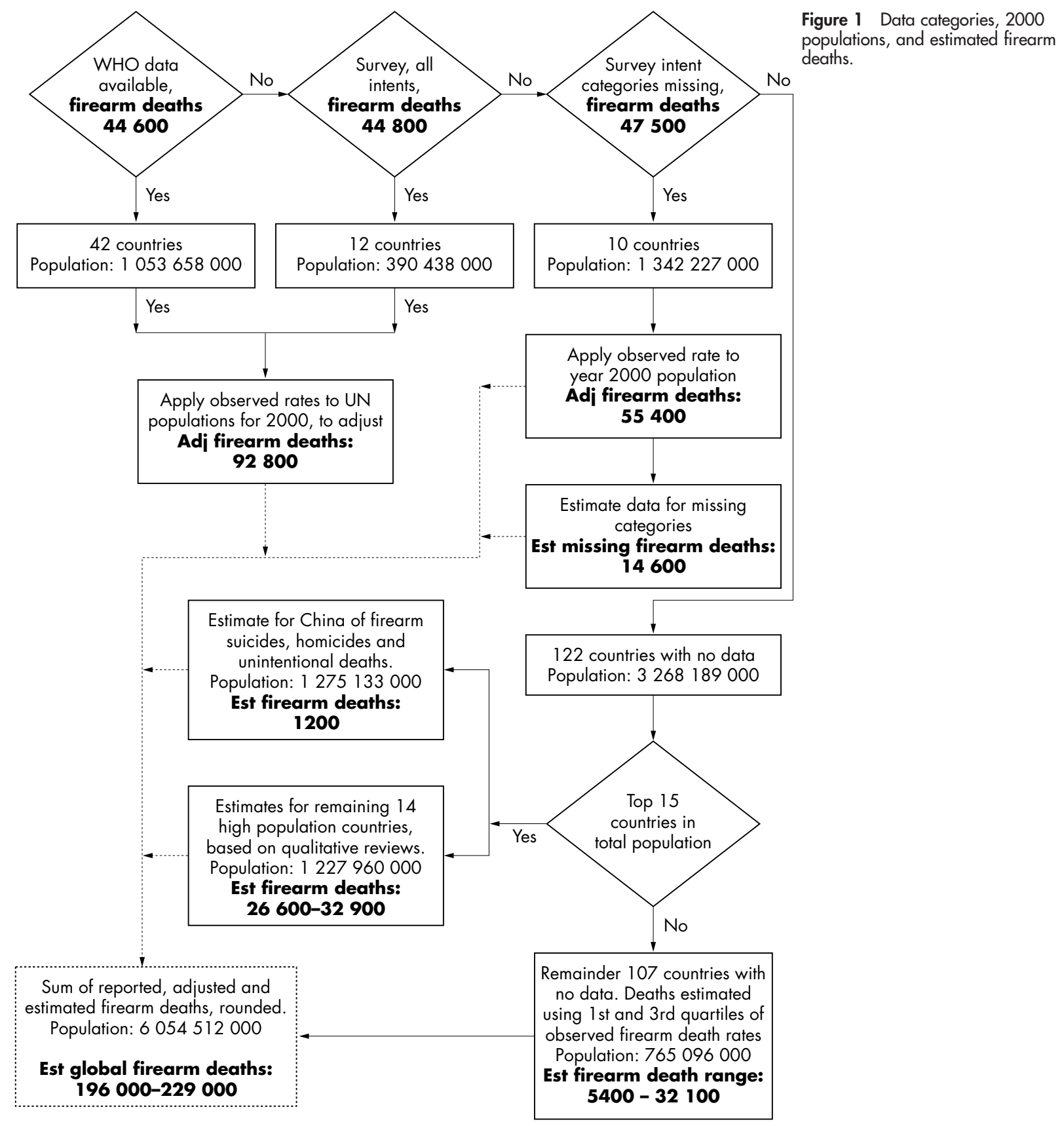

\section{Estimates for countries with FDD}

We projected deaths to the year 2000 for the 64 countries with available data using observed CFDRs. We adjusted total FDD, by using observed ratios of firearm deaths between intent categories from countries with complete data to solve for missing categories in the 10 (of 64) countries with incomplete intent categories. An average unintentional and undetermined CFDR of 0.36 per 100000 was used to estimate these missing deaths.

\section{Estimates for countries without FDD}

We explored several methods for estimating firearm mortality for the 122 countries without FDD. As others have used region and/or income level to build global estimates, ${ }^{11}$ we examined available data by region and economic development level, using World Bank categories. ${ }^{12}{ }^{13}$ We found wide variation in firearm death rates within and between economic levels and regions, and small cell sizes within some region/economic classes. Populations without reported FDD are disproportionately located in lower middle/lower income countries (see http://www.injuryprevention.com/ supplemental for economic level and region data) Therefore, we chose to use data from a number of sources to calculate a global estimate.

Two approaches were used: qualitative literature review and estimation for the 15 most populous countries without FDD (with China handled separately), and application of high and low quartile death rates for the remaining 107 countries.

The literature review used electronic search engines, followed by hand searches of bibliographic references or web page content. Local vital statistics data, allowing the computation of rates, were considered best, though not nationally representative. Death review data (for example, 
Table 1 Fifteen most populous countries and reported firearm deaths

\begin{tabular}{|c|c|c|c|}
\hline $\begin{array}{l}\text { Most populous countries with FDD } \\
\text { (firearm death data year) }^{\text {source }}\end{array}$ & $\begin{array}{l}\text { CFDR/ } 100000 \\
\text { population }\end{array}$ & $\begin{array}{l}\text { Reported firearm deaths, } \\
\text { projected to year } 2000 \\
\text { population* }\end{array}$ & $\begin{array}{l}\% \text { of intentional } \\
\text { deaths by firearm }\end{array}$ \\
\hline India $(1994)^{\mathrm{s} 2}$ & 0.3 & $3300^{*}$ & - \\
\hline United States (1998) ${ }^{\mathrm{w}}$ & 10.9 & 30900 & 63.0 \\
\hline Brazil (1995) ${ }^{\mathrm{s} 1}$ & 26.7 & 45500 & 96.5 \\
\hline Japan $(1997)^{w}$ & 0.1 & 100 & 0.0 \\
\hline Mexico $(1994)^{\mathrm{s} 2}$ & 12.1 & $12000^{*}$ & 61.3 \\
\hline Germany (1999)w & 1.5 & 1200 & 12.7 \\
\hline Viet Nam $(1995)^{\text {s1 }}$ & 0.2 & 100 & - \\
\hline Philippines $(1996)^{\mathrm{s} 2}$ & 3.6 & $2700^{*}$ & 22.8 \\
\hline Thailand (1994) w & 4.3 & 2700 & 36.7 \\
\hline United Kingdom (1999)w & 0.3 & 200 & 4.4 \\
\hline France $(1998)^{\mathrm{w}}$ & 5.0 & 3000 & 32.5 \\
\hline Italy $(1997)^{w}$ & 2.0 & 1200 & 27.9 \\
\hline Republic of Korea (1997)w & 0.1 & 100 & 0.9 \\
\hline South Africa $(1995)^{\text {s2 }}$ & 27.0 & $11700^{*}$ & - \\
\hline Colombia $(1995)^{\mathrm{s} 2}$ & 51.9 & $21800^{*}$ & 79.8 \\
\hline Total & 5.9 & 136400 & 56.9 \\
\hline
\end{tabular}

autopsies, trauma data) have significant biases, but help estimate the proportion of firearm deaths. Other data sources (for example, key informant estimates; community survey) provide only broad indications of firearm deaths.

China was treated separately from the other populous countries without FDD, based on evidence of extremely low rates coupled with a large population. A review of suicide studies for select areas in China ${ }^{14}$ provides an estimate of $0.56 \%$ of suicides by firearm, which could be applied to reported suicide death rates for selected urban and rural areas of China (13.7/100 000). ${ }^{3}$ Linear regression was used to predict percent homicides by firearm ( $95 \%$ CI 0.54 to $0.97 \%$ ), based on observed percent firearm for homicides and suicides among countries with FDD, and applied to reported homicide rates for selected urban/rural areas of China (1.8/100 000). ${ }^{3}$ Unintentional/underdetermined firearm deaths were estimated as $5.7 \%$ of all intentional firearm deaths, based on the ratio observed in the 54 countries reporting all intent categories.

For the remaining 14 most populous countries without data, the literature review was used to classify countries to low, medium, or high firearm mortality rates. As the distribution of the firearm death rates for the 54 countries with complete FDD was strongly skewed, we used the first
$(0.7 / 100000)$, second $(2.0 / 100000)$, or third quartiles $(4.2 /$ $100000)$ to quantify estimates of low, medium, or high firearm death rates, rather than the mean. Four of the most populous countries without FDD had areas of armed conflicts (Indonesia, Democratic Republic of Congo, Nigeria, Sudan). We did not attempt to estimate conflict related firearm deaths, but studies of civilian-on-civilian injuries indicate that firearm mortality in these countries is likely to be high. For the three of these 15 countries without qualitative evidence (Egypt, Ethiopia, Myanmar) and the remaining 107 countries without FDD, first and third quartiles were applied to their year 2000 population.

\section{RESULTS}

The global burden of non-conflict related firearm mortality is estimated at 196000 to 229000 per year (fig 1). Firearm mortality for countries with WHO-WRVH vital statistics and from published surveys of government officials, once adjusted to the year 2000, yielded 92800 deaths, covering $23.8 \%$ of the world's population. Adding adjusted survey data from the 10 additional countries with missing intent categories added another 70000 firearm deaths. In total, these three data sources yielded 162800 firearm deaths for the year 2000 and represent 35\% (64/186 countries) of

Table 2 Fifteen most populous countries not reporting firearm deaths

\begin{tabular}{lrll}
\hline $\begin{array}{l}\text { Most populous countries } \\
\text { without FDD }\end{array}$ & Population & $\begin{array}{l}\text { Estimated* CFDR/100000 } \\
\text { based on qualitative } \\
\text { information }\end{array}$ & $\begin{array}{l}\text { Estimated firearm deaths, } \\
\text { based on qualitative } \\
\text { information }\end{array}$ \\
\hline China & 1275133000 & 0.1 & 1200 \\
Indonesia & 212092000 & 0.7 & 1500 \\
Russian Federation & 145491000 & 0.7 & 3000 \\
Pakistan & 141256000 & 4.2 & 5900 \\
Bangladesh & 137439000 & 4.2 & 5800 \\
Nigeria & 113862000 & 2.0 & 2300 \\
Iran & 70330000 & 0.7 & 500 \\
Egypt & 67884000 & $0.7-4.2$ & $500-2900$ \\
Turkey & 66668000 & 2.0 & 1400 \\
Ethiopia & 62908000 & $0.7-4.2$ & $400-2600$ \\
Democratic Republic of Congo & 50948000 & 4.2 & 2100 \\
Ukraine & 49568000 & 0.7 & 300 \\
Myanmar & 47749000 & $0.7-4.2$ & $300-2000$ \\
Sudan & 31095000 & 4.2 & 1300 \\
Kenya & 30669000 & 4.2 & 1300 \\
Total & 2503093000 & & $27800-34100$ \\
\hline Extremely low =0.1; low (Q1) $=0.7 ;$ medium (Q2) & $=2.0 ;$ high (Q3) =4.2. & \\
\hline
\end{tabular}


potential reporting entities and $46 \%$ of the world's population.

\section{Countries reporting FDD}

The 15 most populous countries reporting FDD cover a population exceeding two billion $(37.9 \%$ of world population), accounting for $92.0 \%$ of reported global firearm deaths (table 1). CFDRs vary substantially. Colombia, South Africa, Brazil, United States, and Mexico have the highest CFDRs and the greatest number of firearm deaths.

\section{Countries not reporting FDD}

The 15 most populous countries not reporting FDD cover a population exceeding 2.6 billion (41.3\% of world population) and represent $76.5 \%$ of the total global population without FDD (15/122 countries) (table 2). The publication review provided empirical evidence of the presence and magnitude of firearm death (see http://www.injuryprevention.com/supplemental for detailed table and sources). Reports based on autopsy, ambulance, and hospital data provided evidence on local firearm injury or deaths. ${ }^{15} 16$ For others, surveys provided indications of firearm violence. ${ }^{17}{ }^{18}$ Evidence for China indicates an extremely low rate, based upon intentional death rates for selected areas ${ }^{19}$ and a meta-review of 13 studies reporting the percent of suicide by firearm. ${ }^{14}$ Our estimates for the 15 most populous countries without data yielded an estimate range of 27800 to 34100 , and our estimates for the remaining 107 countries without data range from 5400 to 32100 .

\section{DISCUSSION \\ Key findings}

The global burden of firearm mortality is estimated to be 196000 to 229000 . This analysis extends beyond international comparisons of firearm mortality typically limited to higher income countries. ${ }^{20-24}$ Missing FDD for many lower income countries and populations in the Middle East, subSaharan Africa, and East Asia, create biases. ${ }^{20}{ }^{21}$ By combining countries with FDD from vital statistics and from other data of varying levels of completeness, our estimate covers substantially more of the world's population.

\section{Study strengths and limitations}

Our approach to informing the estimates of the 15 most populous countries without FDD incorporated new data from a variety of sources. As these countries represent $76.5 \%$ of the populations without FDD, published literature for these countries is an important resource. ${ }^{625}$ Readers can easily update our global estimate as new data become available. Our estimates used publicly available health data and publications or abstracts available in English. Although it is difficult to validate our approach, our similar yet tighter estimates than the Small Arms Survey findings lends credibility to our estimations. ${ }^{11}$ The Small Arms Survey provides an estimate of $200000-270000$ with some differences in data sources and analytic techniques. ${ }^{11}$

Combining available sources and making estimates for missing data provide more comprehensive population coverage, at the expense of some precision. Even with reported FDD, incomplete death or population coverage can result in under or overreporting for regions (for example, rural) or people (for example, stigmatized deaths or marginalized groups). Survey responses may be biased for countries with greater interest in firearm deaths. The effects of underreporting in published data may downward bias both reported and estimated deaths.

Projecting reported FDD rates to the year 2000 population assumes a constant rate of firearm death, although temporal variations are to be expected. Recent data, external to our dataset, illustrate the potential impact. For example, United States data indicate approximately 2000 fewer deaths than our estimate, while qualitative evidence indicates increasing firearm deaths in some countries without data (for example, Russian Federation). ${ }^{726}$ A recent report on firearm deaths in Brazil identifies changes in data quality, which suggests lower estimates for year 2000 firearm deaths than reported here. ${ }^{27}$

\section{Conflict related mortality}

We excluded conflict related mortality (as distinct ICD codes) in this analysis. Yet conflict affects non-conflict related firearm deaths, which can increase with the influx and residual presence of firearms. ${ }^{28}$ Military weapons can move rapidly into civilian sectors and illegal transport, importation, and availability of firearms is a problem on all continents. ${ }^{10}{ }^{29}$ Evidence suggests that small arms left behind from conflicts do cause injuries in countries without FDD. ${ }^{30-34}$

\section{Recommendations}

Three major recommendations stem from this study: improve data, recognize the burden of firearm mortality, and take public health action. Improving surveillance, data availability, and specificity are important; however this requires government and social stability, financial investment, infrastructure, and human resource commitment. ${ }^{35}{ }^{36}$ Proper classification of deaths from firearm violence requires more complex systems, incorporating both mechanism and intent. Where government vital statistics collection is not feasible, surveillance or descriptive data from other sources become increasingly important. Adding other approaches to traditional surveillance systems-such as surveys, hospital and emergency transport data, and humanitarian aid and mortuary data-has much to offer. ${ }^{14} 15192836$ Although limited in scope, these types of data can help identify trends in firearm injury and death and better portray the local and regional burden.

The local and global health burden of firearm mortality is clear and compelling. Over the next two decades the absolute number of firearm deaths will increase as populations at risk continue to grow, particularly in lower income countries without FDD. Global demographic shifts in urbanization and poverty could increase risks for firearm violence. ${ }^{1}$ In addition, the worldwide proliferation of small arms and their diffusion into civilian populations seem to make this escalation of global firearm violence inevitable. ${ }^{30} 31$

Public health action is important, although the science on effective prevention programs is limited. ${ }^{37}$ Building a better empirical foundation for addressing the sociocultural and economic environments that enhance or mitigate the potential for firearm death should be an international effort. The high variability of firearm death rates provides a valuable opportunity to use cross national comparisons to explore and better understand risk factors. ${ }^{38}$ Analysis of the effects of firearm availability and legislative approaches to firearm violence on the health of citizens are often limited to countries with FDD. ${ }^{23} 2439$ These policies include firearm and ammunition designs, manufacturing and distribution, access to firearms (legal limitations and strategies to address firearms left behind in regional conflict), import and export controls, and offender access. Such information and experience could guide the world health community and individual countries in developing effective responses to firearm injury.

Firearm related mortality must be viewed as a health problem of substantial burden, which extends beyond national borders and is dynamic in nature. The world health community can take action to improve global understanding and make policy recommendations that begin to address the complex series of events that result in firearm injury. 


\section{Key points}

- Global non-conflict firearm deaths are estimated to be 196000 to 229000.

- Countries with the most complete firearm data covered only $23.8 \%$ of the world's population.

- The distribution of populations without reported firearm death data are disproportionately located in lower middle and lower income countries.

- Total firearm deaths for the 15 most populous countries without reported data are estimated to be 27800 to 34100 deaths per year.

\section{ACKNOWLEDGEMENTS}

We acknowledge James A Mercy PhD for his thoughtful review of an earlier version of this paper.

\section{Authors' affiliations}

T S Richmond, School of Nursing, Firearm and Injury Center at Penn, Senior Fellow, Leonard Davis Institute of Health Economics, University of Pennsylvania, PA, USA

R Cheney, Firearm and Injury Center at Penn, University of

Pennsylvania, PA, USA

C W Schwab, Division of Traumatology \& Surgical Critical Care, School of Medicine, Firearm and Injury Center at Penn, University of Pennsylvania, PA, USA

Funding source: the Joyce Foundation located in Chicago, IL provided financial support for the Firearm \& Injury Center at Penn (FICAP). The funder had no role in the conduct of the study, the interpretation of the data, or in the preparation and review of this manuscript.

Competing interests: none.

All authors had full access to all data in the study and had full final responsibility for the decision to submit this manuscript for publication.

Variations of this paper were presented at: Global Health Council Meeting, 2003, the Population Association of America Meeting, 2004, and the 7th World Injury Conference, 2004.

\section{REFERENCES}

1 Howson CP, Fineberg HV, Bloom BR. The pursuit of global health: The relevance of engagement of developed countries. Lancet 1998;351:586-90.

2 Walt G. Globalisation of international health. Lancet 1998;351:434-7.

3 Krug EG, Dahlberg LL, Mercy JA, et al. World report on violence and health. Geneva: World Health Organization, 2002.

4 United Nations. The seventh United Nations survey on crime trends and the operations of criminal justice systems (1998-2000), United Nations Office of Drugs and Crime; Table 2.04.[Online]; 2003.Available at http:// www.unodc.org/unodc/crime_cicp_survey_seventh.html (accessed March 2004).

5 Centers for Disease Control and Prevention. Web-based statistics query and reporting system (WISQARS) [Online]. National Center for Injury Prevention and Control, CDC (producer); 2003. Available at www.cdc.gov/ncipc/ wisquars (accessed March 2004).

6 Krug EG, Powell KE, Dahlberg LL. Firearm-related deaths in the United States and 35 other high- and upper-middle-income countries. Int J Epidemiol 1998;27:214-21

7 Beaglehole R, Bonita R. Public health at the crossroads: Which way forward? Lancet 1998;351:590-2.
8 Cukier W. Firearms regulation: Canada in the international context. Chronic Dis Can 1998; 19:25-34.

9 United Nations. Total population by sex and sex ratio, by country, 2000 medium-variant, Table 1-2000, Available at http://www.un.org/esa/ population/publications/wpp2000/annex-tables.xls (accessed November 2004)

10 United Nations. United Nations international study on firearm regulation. New York: United Nations, 1998.

11 Small Arms Survey. Small arms survey 2004. Oxford: Oxford University Press, 2004.

12 World Bank. World Development Report 2003: Sustainable development in a dynamic world. Oxford: Oxford University Press, 2002.

13 World Bank. List of economies, 2003. Available at: http:// www.worldbank.org/data/countryclass/history.htm (accessed April 2003).

14 He ZX, Lester D. Methods for suicide in mainland China. Death Stud 1998;22:571-9.

15 Chotani H, Razzak J, Luby S. Patterns of violence in Karachi, Pakistan. Inj Prev 2002;8:57-9.

16 Goren S, Subas M, Tirasci Y. Firearm-related mortality: A review of four hundred forty four Deaths in Diyarbakir, Turkey between 1996 and 2001. Tohoku Journal of Experimental Med 2001;201:139-45.

17 UN Small Arms Survey. Caught in the crossfire: the humanitarian impacts of small arms. Available at http://www.reliefweb.int/library/documents/ 2002/giis-con-30jul.pdf 2002: 155-99 (accessed November 2004).

18 Depoortere E, Checchi F, Briollet F, et al. Violence and mortality in West Darfu, Sudan (2003-2004) epidemiologic evidence from four surveys. Lancet 2004; 364:1315-20

19 Zhao Y, Wang W. Neurosurgical trauma in People's Republic of China. World J Surg 2001;25:1202-4.

20 Centers for Disease Control. Rates of homicide, suicide and firearm-related death among children -26 industrialized countries. MMWR Morb Mortal Wkly Rep 1997;46:101-6.

21 Fingerhut L, Cox CS, Warner M. International comparative analysis of injury mortality: findings from the ICE on injury statistics. Adv Data 1998;303:1-19, Hyattesville, MD, National Center for Health Statistics.

22 UNICEF. A league of child deaths by injury in rich nations. Innocenti Report Card No 2. Florence: UNICEF Innocenti Research Centre, 2001

23 Hemenway D, Miller M. Firearm availability and homicide rates across 26 high-income countries. J Trauma 2000;49:985-8.

24 Hemenway D, Shinoda-Tagawa T, Miller M. Firearm availability and female homicide victimization rates among 25 populous high-income countries. J Am Med Womens Assoc 2002;57:100-4.

25 Forjuoh SN, Zwi, AB, Mock CN. Injury control in Africa: getting governments to do more. Trop Med Int Health 1998;3:349.

26 Russian council gives figures for crime with guns, bombs. Available at http:// www.nisat.org/default.asp?page = / search.asp April 24, 2000 Document \#FBIS-SOV-2000-0425 (accessed March 2005).

27 Centers for Disease Control and Prevention. Homicide trends and characteristics-Brazil, 1980-2002. MMWR Morb Mortal Wkly Rep, 2004;53:169-71

28 Cukier W. Small arms and light weapons: a public health approach. The Brown Journal of World Affairs 2002;IX:261-80.

29 Muggah R, Berman E. Humanitarianism under threat: the humanitarian impacts of small arms and light weapons. Geneva: Small Arms Survey, 2001

30 Beri R. Coping with small arms threat in South Africa. Strategic Analysis: A Monthly J of the IDSA 2000;XXIV(1)

31 Conrad B. The problem of small arms and light weapons in Taiikistan. Strategic Analysis: A Monthly J of the IDSA 2002;24(8)

32 Hartung WD. The new business of war: small arms and the proliferation of conflict. Ethics Int Aff 2001;15:79-92.

33 Rawson B. Aiming for prevention: Medical and public health approaches to small arms, gun violence, and injury. Croat Med J 2002;43:379-85.

34 Lacey M. Kenya burns weapons in anticrime crackdown. NY Times 2003. Available at http://www.nytimes.com/2003/03/16/international/africa/ 16Keny.html (accessed November 2004).

35 Meddings D. Aiming for prevention. Presentation at International Medical Conference on Small Arms, Gun Violence, \& Injury. Helsinki, Finland, September 28-30, 2001.

36 Meddings $\mathbf{D}$. The value of credible data from under-resourced areas. Med Confl Surviv 2003;18:380-8.

37 In: Wellford CF, Pepper JV, Petrie CV, eds. Firearm and violence: a critical review, Washington DC, 2004

38 Killias M, van Kesteren J, Rindlisbacher M. Guns, violent crime, and suicide in 21 countries. Can J Criminol 2001;43:429-48.

39 Cukier W, Sarker T, Quigley T. Firearm regulation: International law and jurisprudence. Canadian Criminal Law Review 2000;6:99-12. 